\title{
EFFECTIVE YOUNG'S MODULUS OF ORTHOTROPIC LAMINATE WITH ORIENTED UNIDIRECTIONAL FIBROUS LAMINAS
}

\author{
M. Kukliński*
}

\begin{abstract}
This report presents the derivation of formula for effective longitudinal Young's modulus of laminate composed of symmetrically oriented, unidirectionally reinforced laminas. Single lamina may be treated as an orthotropic layer when the directions of unidirectional reinforcement and tensile loading are the same. With orientation angle $\theta$ other than a multiple of $\pi / 2$ the lamina becomes a monoclinic layer. Nevertheless, when the symmetrical sequence of oriented laminas is considered e.g. $\theta /-\theta /-\theta / \theta$, they constitute an orthotropic laminate as a whole. It is obvious that the effective Young's moduli of single monoclinic lamina and the resultant symmetrical orthotropic laminate should be different and they are, as it is shown in this report. For comparison the material constants of glass-polyester laminate have been applied.
\end{abstract}

Keywords: effective Young's modulus, orthotropic laminate, monoclinic laminate, fibrous composites

\section{Introduction}

Researches into effective Young's modulus of multiple phase materials have well documented tradition. In the $19^{\text {th }}$ century $\mathrm{W}$. Voigt (1889) presented the effective stiffness matrix of two phase composite material for assumed isostrain model of deformation. A few decades later A. Reuss (1929) derived and published the effective compliance matrix for composite under isostress condition. As Voigt and Reuss did not include the influence of Poisson's ratio on effective Young's modulus of composite, their models are limited only to one-dimensional case. The compliance matrix including Poisson effect of composite composed of isotropic phases may be found in publication of Bin Liu et al. (2006). The analysis of fibre orientation influence on Young's modulus for unidirectionally reinforced laminate presented H.W. Wang et al. (2014).

This work constitutes an extension of the cited above researches onto orthotropic laminates composed of monoclinic layers. Obtained results have been compared with Voigt model (Eq. 1), which generally underestimates the resultant effective longitudinal Young's modulus.

$$
E_{\text {eff }}^{\text {Voigt }}=\Phi_{A} \cdot E_{A}+\Phi_{B} \cdot E_{B}
$$

where:

$\Phi_{A}, E_{A}$ - respectively volume fraction and Young's modulus of phase $A$,

$\Phi_{B}, E_{B}$ - respectively volume fraction and Young's modulus of phase $B$,

As the layers of the considered laminate have the same structure and the laminate is orthotropic, then it follows that $\Phi_{A}=\Phi_{\theta}=0.5$ and $\Phi_{B}=\Phi_{-\theta}=0.5$, where $\theta$ is the angle of orientation relative to the tension direction. The layers are symmetrically oriented at the same angle but in opposite directions as shown in Fig. 1. In consequence, although each oriented layer is monoclinic, the resultant laminate is orthotropic.

Mariusz Kuklinski, PhD.: Faculty of Mechanical Engineering, UTP University of Science and Technology in Bydgoszcz, Al. prof. S. Kaliskiego 7, 85-796 Bydgoszcz, PL, mariusz.kuklinski@utp.edu.pl 

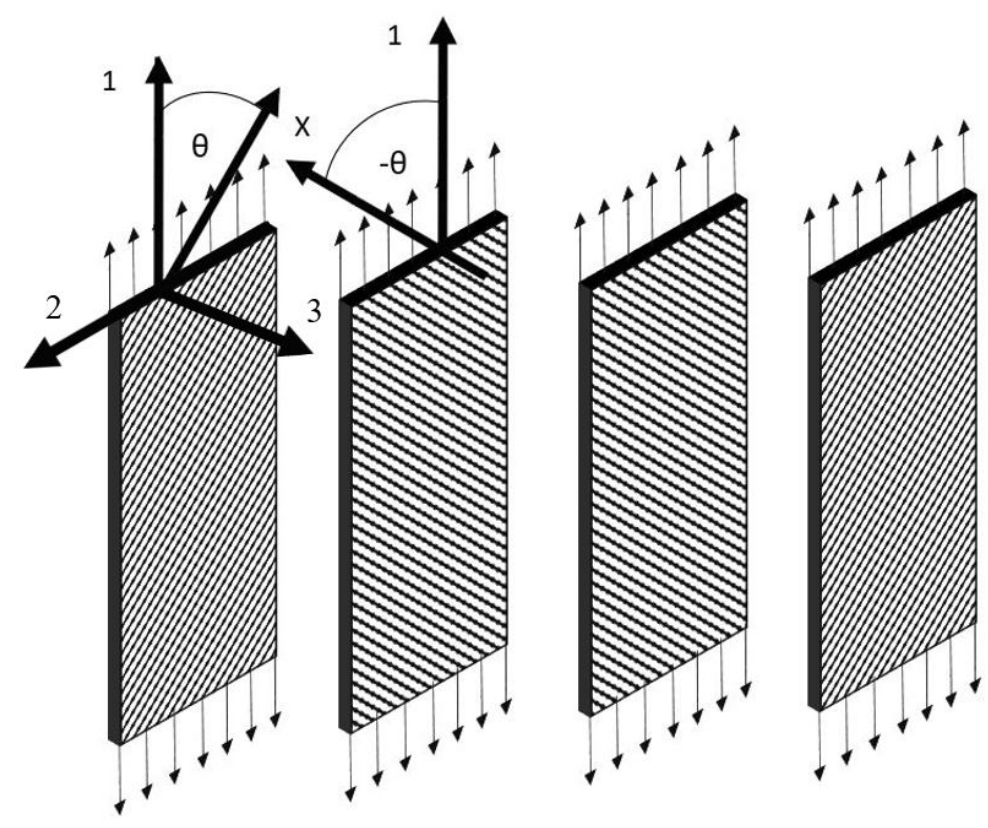

Fig. 1: Exemplary sequence of symmetrically oriented laminas.

Each layer with reinforcement oriented along axis $X$ (Fig. 1) is orthotropic in $X Y Z$ coordinate system, but after rotation at angle $\theta$ becomes a monoclinic layer in 123 coordinate system. Axes $Z$ and 3 coincide in chosen coordinate systems. The compliance matrix $\mathbf{S}$ of orthotropic layer is given in Eq. 2.

$$
\left\{\begin{array}{l}
\varepsilon_{x x} \\
\varepsilon_{y y} \\
\varepsilon_{z z} \\
\gamma_{y z} \\
\gamma_{x z} \\
\gamma_{x y}
\end{array}\right\}=\left[\begin{array}{cccccc}
\frac{1}{E_{x}} & \frac{-v_{y x}}{E_{y}} & \frac{-v_{z x}}{E_{z}} & & & \\
\frac{-v_{x y}}{E_{x}} & \frac{1}{E_{y}} & \frac{-v_{z y}}{E_{z}} & 0 & 0 & 0 \\
\frac{-v_{x z}}{E_{x}} & \frac{-v_{y z}}{E_{y}} & \frac{1}{E_{z}} & 0 & 0 & 0 \\
& & & \frac{1}{G_{y z}} & 0 & 0 \\
0 & 0 & 0 & 0 & \frac{1}{G_{x z}} & 0 \\
0 & 0 & 0 & 0 & 0 & \frac{1}{G_{x y}} \\
0 & 0 & 0 & 0 & \\
& & & & &
\end{array}\right] \cdot\left\{\begin{array}{l}
\sigma_{x x} \\
\sigma_{y y} \\
\sigma_{z z} \\
\tau_{y z} \\
\tau_{x z} \\
\tau_{x y}
\end{array}\right\} \Rightarrow \boldsymbol{\varepsilon}=\boldsymbol{S} \cdot \boldsymbol{\sigma}
$$

After rotation at angle $\theta$ the compliance matrix $\overline{\boldsymbol{S}}$ of lamina takes the form given in Eq. 3 .

$$
\left\{\begin{array}{l}
\varepsilon_{1} \\
\varepsilon_{2} \\
\varepsilon_{3} \\
\varepsilon_{4} \\
\varepsilon_{5} \\
\varepsilon_{6}
\end{array}\right\}=\left[\begin{array}{cccccc}
s_{11} & s_{12} & s_{13} & 0 & 0 & s_{16} \\
s_{21} & s_{22} & s_{23} & 0 & 0 & s_{26} \\
s_{31} & s_{32} & s_{33} & 0 & 0 & s_{36} \\
0 & 0 & 0 & s_{44} & s_{45} & 0 \\
0 & 0 & 0 & s_{54} & s_{55} & 0 \\
s_{61} & s_{62} & s_{63} & 0 & 0 & s_{66}
\end{array}\right] \cdot\left\{\begin{array}{l}
\sigma_{1} \\
\sigma_{2} \\
\sigma_{3} \\
\sigma_{4} \\
\sigma_{5} \\
\sigma_{6}
\end{array}\right\} \Rightarrow \overline{\boldsymbol{\varepsilon}}=\overline{\boldsymbol{S}} \cdot \overline{\boldsymbol{\sigma}}
$$

\section{Derivation of effective Young's modulus of considered laminate}

As shown in Fig. 1, the axial tension in direction 1 is considered. In derivation of the formula for effective longitudinal Young's modulus, assuming that $\Phi_{\theta}=\Phi_{-\theta}=0.5$, the following relations are essential:

- resultant stress in laminate is the sum of products of stress and volume fraction in each lamina:

$$
\begin{aligned}
& \sigma_{1}=0.5\left(\sigma_{1}^{A}+\sigma_{1}^{B}\right) \\
& \sigma_{2}=0.5\left(\sigma_{2}^{A}+\sigma_{2}^{B}\right) \\
& \sigma_{6}=0.5\left(\sigma_{6}^{A}+\sigma_{6}^{B}\right)
\end{aligned}
$$

- strains in layer's plane are the same in each lamina: 


$$
\begin{aligned}
& \varepsilon_{1}=\varepsilon_{1}^{A}=\epsilon_{1}^{B} \\
& \varepsilon_{2}=\varepsilon_{2}^{A}=\epsilon_{2}^{B} \\
& \varepsilon_{6}=\varepsilon_{6}^{A}=\epsilon_{6}^{B}
\end{aligned}
$$

- assumed boundary conditions are:

$$
\sigma_{3}=\sigma_{3}^{A}=\sigma_{3}^{B}=0 ; \quad \sigma_{2}=0 ; \quad \sigma_{6}=\tau_{12}=0
$$

The superscripts $A, B$ refer to laminas oriented respectively at angle $\theta$ and $-\theta$. The resultant stresses $\sigma_{2}$ and $\tau_{12}=\sigma_{6}$ in laminate as a whole equal zero, then Eqs. 5-6 yield:

$$
\begin{aligned}
& \sigma_{2}^{A}=-\sigma_{2}^{B} \\
& \sigma_{6}^{A}=-\sigma_{6}^{B}
\end{aligned}
$$

As the volume fractions and absolute values of orientation angles are the same for both phases $A$ and $B$, it follows that:

$$
\sigma_{1}^{A}=\sigma_{1}^{B}=\sigma_{1}
$$

The effective longitudinal Young's modulus of laminate may be calculated from the Eq. 14.

$$
E_{1}^{e f f}=\frac{\sigma_{1}}{\varepsilon_{1}^{A}}=\frac{\sigma_{1}}{\varepsilon_{1}^{B}}
$$

From Eqs. 2, 3 and 11-13 the lamina's strains have been derived and take form of Eqs. 15-16.

$$
\begin{aligned}
& \varepsilon_{1}^{A}=\sigma_{1} \cdot s_{11}^{A}+\sigma_{2}^{A} \cdot s_{12}^{A}+\sigma_{6}^{A} \cdot s_{16}^{A} \\
& \varepsilon_{1}^{B}=\sigma_{1} \cdot s_{11}^{B}-\sigma_{2}^{A} \cdot s_{12}^{B}-\sigma_{6}^{A} \cdot s_{16}^{B}
\end{aligned}
$$

Noting that $\mathrm{s}^{\mathrm{A}}{ }_{11}=\mathrm{s}^{\mathrm{B}}{ }_{11}, \mathrm{~s}^{\mathrm{A}}{ }_{12}=\mathrm{s}^{\mathrm{B}}{ }_{12}, \mathrm{~s}^{\mathrm{A}}{ }_{16}=-\mathrm{s}^{\mathrm{B}}{ }_{16}$ and substituting Eqs. 15, 16 into Eq. 7 yields:

$$
\sigma_{2}^{A}=\sigma_{2}^{B}=0
$$

Noting that $s_{66}^{\mathrm{A}}=\mathrm{s}_{66}^{\mathrm{B}}$, from Eqs. 3, 9, 12 and 17 the stress $\sigma_{6}{ }^{\mathrm{A}}$ has been calculated (Eq. 18).

$$
\sigma_{6}^{A}=-\sigma_{1} \cdot \frac{s_{61}^{A}}{s_{66}^{A}}
$$

Substituting Eqs. 15, 17 and 18 into 14 the formula for effective longitudinal Young's modulus has been derived in the form of Eq. 19.

$$
\frac{1}{E_{1}^{e f f}}=s_{11}-\frac{\left(s_{16}\right)^{2}}{s_{66}}
$$

In derivation process the following elements of oriented layer's compliance matrix (Eq. 3), presented also by Reddy (2004), have been applied:

$$
\begin{gathered}
s_{11}=\frac{1}{E_{x}} \cos ^{4} \theta+\left(\frac{1}{G_{x y}}-2 \frac{v_{x y}}{E_{x}}\right) \cos ^{2} \theta \cdot \sin ^{2} \theta+\sin ^{4} \theta \frac{1}{E_{y}} \\
s_{12}=\frac{-v_{x y}}{E_{x}} \cos ^{4} \theta+\left(\frac{1}{E_{x}}+\frac{1}{E_{y}}-\frac{1}{G_{x y}}\right) \cos ^{2} \theta \cdot \sin ^{2} \theta-\sin ^{4} \theta \frac{v_{x y}}{E_{x}} \\
s_{16}=\left(\frac{2}{E_{x}}+\frac{2 v_{x y}}{E_{x}}-\frac{1}{G_{x y}}\right) \cos ^{3} \theta \cdot \sin \theta+\left(\frac{1}{G_{x y}}-\frac{2 v_{x y}}{E_{x}}-\frac{2}{E_{y}}\right) \sin ^{3} \theta \cdot \cos \theta \\
S_{66}=\frac{\left(\cos ^{2} \theta-\sin ^{2} \theta\right)}{G_{x y}}+4\left(\frac{1}{E_{x}}+\frac{1}{E_{y}}+\frac{2 v_{x y}}{E_{x}}\right) \cos ^{2} \theta \cdot \sin ^{2} \theta
\end{gathered}
$$

The one important conclusion follows from Eq. 19: the effective Young's modulus of considered laminate is not less than that of a single rotated lamina i.e.:

$$
E_{1}^{e f f} \geq \frac{1}{s_{11}}
$$

In Eq. 24 the equality occurs only when orientation angle $\theta=n \cdot \pi / 2, n$ being a natural number or for $\theta=$ $50^{\circ}$, as seen in Fig. 2. The graphical comparison of the derived $E_{1}{ }^{e f f}$ with the inverse of $s_{11}$ is shown in Fig. 2. The material constants of unidirectionally reinforced glass-polyester laminate have been adopted in the graph: $\mathrm{E}_{1}=34533 \mathrm{MPa}$ and $\mathrm{E}_{2}=10035 \mathrm{MPa}$. The differences are noticeable. The resultant Young's modulus of laminate is much greater than that of a single oriented lamina as the effect of mutual 
stiffening of laminate's oriented layers. As seen in Fig. 2 at some angles the value of effective Young's modulus is even twice that of a single layer.

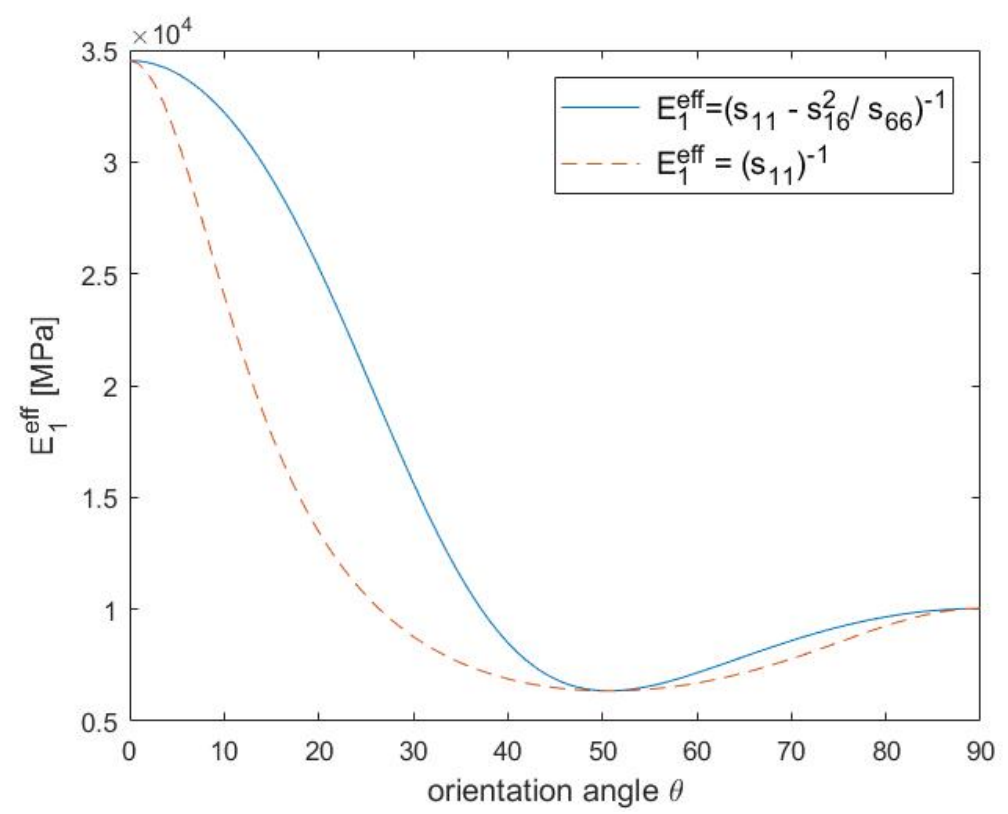

Fig. 2: Derived effective Young's modulus and inverse of $s_{11}$ as function of the orientation angle $\theta$.

\section{Conclusions}

This report contains the derivation of the effective longitudinal Young's modulus of orthotropic laminate composed of symmetrically oriented, unidirectionally reinforced laminas. The derived formula may be very useful in construction of yacht's hulls. It gives a latitude in designing the desirable stiffness of a hull in specified directions by substituting heavy multi-angle glass fabrics (e.g. -45/0/45) by unidirectional one applied at specified angles. This gives the possibility of building more lightweight and durable constructions.

The derived formula (Eq. 19) applies to laminate composed of identical single unidirectional fibrous layers, oriented at angles of the same absolute values, with equal volume fractions $\left(\Phi_{A}=\Phi_{B}=0.5\right)$ and symmetrical arrangement relative to the neutral plane of laminate, parallel to the plane 12 in Fig. 1. Exemplary laminate structure may be: $-\theta / \theta / \theta /-\theta$.

The graph in Fig. 2 shows that the effect of mutual stiffening of laminate's oriented layers is significant and effective Young's modulus of laminate, in some ranges of orientation angle $\theta$, is much greater than that of the single layer.

The next step of research into presented aspect of fibrous composites may be the derivation of formulae for all plain-stress-state material constants and subsequently an extension of the scope of application of formulae to any layered composite.

\section{References}

Bin Liu, Lixian Zhang, Huajian Gao (2006) Poisson ratio can play a crucial role in mechanical properties of biocomposites. Mechanics of Materials, 38, pp. 1128-1142.

Reddy J. N. (2004) Mechanics of laminated composite plates and shells: theory and analysis. Boca Raton, Florida.

Reuss, A. (1929) Berchung der Fiessgrenze von Mischkristallen auf Grund derPlastiziatsbedingung fur Einkristalle. Zeitschrift für Angewandte Mathematik und Mechanik, 09, pp. 49-58.

Voigt, W. (1889) Uber die Beziehung Zwischen Den Beiden Elastiziatskonstanten Isotroper Korper. Wied Ann, 38, pp. 573-87.

Wang, H.W., Zhou H.W., Gui L.L., Ji H.W., Zhang, X.C. (2014) Analysis of effect of fiber orientation on Young's modulus for unidirectional fiber reinforced composites. Composites: Part B, 56, pp. 733-739. 